\title{
Actualización
}

\section{Manejo de la Osteoporosis: Prevención y Tratamiento}

En grupos de población definidos por la edad y por otros factores de riesgo, la disminución de la masa ósea puede condicionar la producción de fracturas cuyo impacto global sobre el estado de salud es considerable. La definición estricta de osteoporosis corresponde a la pérdida de masa osea que determina fracturas. La masa ósea medida a través de la densitometría (DMO) se reporta como el score $T$, que representa una comparación respecto de la masa ósea de una mujer normal premenopáusica en unidades de desvío estandard (DE). Esto define los diagnósticos operativos de osteopenia cuando los valores se encuentran entre -1 y -2.5 DE y de osteoporosis cuando son iguales o inferiores a $-2.5 \mathrm{DE}$.

Por otro lado hay factores de riesgo independientes de los valores de la densitometría osea que son predictores de fractura como los antecedentes personales de fractura, el bajo peso (menos de $52.2 \mathrm{~kg}$ ), los antecedentes familiares de fractura y el tabaquismo. De tal manera hay situaciones en las que se decide instaurar tratamiento, aún con valores no críticos de densitometría (ej. paciente con antecedentes familiares y personales de osteoporosis con un score mayor a 2.5 en la que se decide el comienzo del tratamiento). Entre las causas de osteoporosis secundaria mencionamos al síndrome de Cushing, el uso crónico de corticoides, el hiperparatiroidismo, el hipogonadismo, las neoplasias, otras drogas, etc.

\section{PREVENCI $\square$ N DE LA OSTEOPOROSIS}

Existen varias estrategias que se describen a continuación.

\section{Terapia de reemplazo hormonal (TRH)}

Existe evidencia proveniente de distintos estudios observacionales que demuestran entre un 35 y un $50 \%$ de reducción del riesgo de fracturas vertebrales y de cadera con el uso de TRH durante 5 a 10 años. Algunos autores sugieren que el tratamiento debería ser instaurado lo más rápidamente posible luego del comienzo del climaterio, si bien varios estudios demostraron un efecto protector, especialmente en la fractura de cadera, en pacientes que comenzaron con TRH a los 60 y 65 años. Esto, a su vez, atenuaría el impacto de la TRH sobre el riesgo de cáncer de mama y útero debido al menor tiempo de exposición.

La mayoría de los autores coinciden en que para que el efecto de la TRH sea beneficioso sobre el hueso, éste debería utilizarse de por vida a una dosis de por lo menos $0.625 \mathrm{mg} /$ día de estrógenos conjugados o su equivalente, ya que se sabe que la suspensión del tratamiento anula inmediatamente su efecto. El agregado de progesterona no altera la eficacia de la TRH.

\section{Reducción del tabaquismo y del consumo de alcohol}

El fumar acelera la pérdida ósea mediante mecanismos que, por ahora, no se conocen. Se estima que las mujeres tabaquistas tienen un $40 \%$ más de riesgo de fractura de cadera y un $30 \%$ más de fractura de columna, con un efecto dosis dependiente según el número de cigarrillos consumidos.

La ingesta moderada de alcohol no afecta la densidad ósea. Sin embargo los alcohólicos muestran una disminución de la misma.

\section{Indicación de actividad física}

Si bien no hay evidencia de estudios randomizados de que disminuya la incidencia de fracturas, el ejercicio aumenta la densidad ósea. Se recomienda que sea variado (reforzando la actividad de los cuádriceps, abdominales y músculos paravertebrales), indicarlo tres veces por semana durante más de 30 minutos por día y con distintas cargas. La actividad física también actúa sobre el otro factor primordial de la prevención de las fracturas: la disminución de las caídas al mejorar el equilibrio.

La inmovilización prolongada (más de tres meses) favorece la resorción ósea y predispone a las fracturas. Estos pacientes, independientemente del momento en que fueron inmovilizados, tendrán a lo largo de toda su vida un riesgo aumentado de sufrir osteoporosis.

\section{Indicación de calcio}

El valor del calcio en la dieta es muy debatido. Sus requerimientos aumentan durante la perimenopausia debido a la disminución de la absorción fraccional de calcio en el intestino. Para preservar la masa cortical, la dosis necesaria en la premenopausia es de $1 \mathrm{~g} /$ día, siendo. en la postmenopausia de 1.2 a 1.5g/día. Sin embargo el agregado de calcio para la prevención de la pérdida ósea no es tan efectivo como la TRH. Un vaso de leche o un yoghurt o una porción de $50 \mathrm{~g}$ de queso aportan $300 \mathrm{mg}$ de calcio cada uno.

Si la paciente no consume en la dieta el equivalente a $1 \mathrm{~g}$ calcio por día se recomienda utilizar un suplemento de calcio en tabletas, siendo el costo y la tolerancia los problemas en esta estrategia ya que suele producir molestias abdominales como pesadez y dolor (las tabletas masticables son las mejor toleradas). El suplemento de calcio se indica lejos de las comidas, lo que mejora su absorción; mientras que en los gerontes, debido a su alta prevalencia de acloridia se recomienda darlo con las comidas y asociado a vitamina $D$ en caso de déficit. Si bien en las mujeres con alto riesgo de fracturas la terapia con calcio no resulta suficiente para reducir su riesgo, siempre debe indicarse una adecuada ingesta cálcica en los pacientes de todas las edades.

\section{Vitamina D}

Actúa sobre los osteoblastos y los osteoclastos e interviene en la absorción del calcio. Los gerontes no la ingieren en suficiente cantidad, se exponen menos al sol y además tienen menos conversión en el riñón a la forma 1-25dihidroxi vitamina $D$, lo que determina un déficit de la forma activa y un aumento de la síntesis de hormona paratiroidea (PTH) produciendo un hiperparatiroidismo secundario que lleva a mayor pérdida ósea.

La evidencia a favor del uso de vitamina D y calcio (como suplemento no dietario) en forma preventiva es contradictoria. Debería ser una indicación formal en los gerontes institucionalizados con déficit de aporte de aquella ( 400 a 800 UI diarias). Debe insistirse en aportar calcio y vitamina $\mathrm{D}$ a través de la dieta y de la exposición al sol. Cuando el aporte es insuficiente, se debe suplementar en forma farmacológica, independientemente del uso de TRH.

\section{TRATAMIENTO DE LA OSTEOPOROSIS}

\section{1) Terapia de reemplazo hormonal}

La TRH sigue siendo uno de los tratamientos de elección ya sea en las pacientes con osteoporosis diagnosticada por densitometría y que no han sufrido fracturas o en quienes si las han padecido.

\section{2) Bifosfonatos}

Los bifosfonatos son análogos sintéticos del pirofosfato, una sustancia natural que inhibe la normal mineralización del hueso. Actúan inhibiendo la resorción ósea. Han sido utilizados en varias enfermedades como el Paget y la hipercalcemia tumoral. La potencia de su efecto antirresortivo depende de los diferentes compuestos, siendo su toxicidad generalmente baja. Han demostrado 
eficacia en el tratamiento de la osteoporosis de columna y hay expectativas favorables en cuanto a su uso en el tratamiento de la de cuello de fémur y en la prevención primaria.

Si bien el tema no está totalmente definido, un efecto interesante de los bifosfonatos es que la masa ósea recuperada parece mantenerse por algunos años.

\section{2-1) Alendronato}

A este grupo de drogas pertenece el alendronato que, utilizado a una dosis de $10 \mathrm{mg}$ por día más el agregado de calcio por un periodo de tres años, demostró disminuir las fracturas de columna vertebral a la mitad ( $R R=0.5)$ y mejorar la densidad ósea de vértebras y huesos largos en mujeres con DMO con Valor T menor a 2. Los metanálisis que evaluaron la eficacia de esta droga han demostrado que disminuye la incidencia de fracturas de cuello de fémur y otros huesos largos. Tendría la ventaja de que se acumula en el hueso y su efecto persistiría varios años (a diferencia de la TRH cuyo efecto sólo se mantiene mientras se utiliza y disminuye al suspenderla). Debe ingerirse en ayunas y acompañado de abundante líquido (se aconseja agua). Sus efectos adversos más frecuentes son las náuseas, los vómitos, la dispepsia, el dolor abdominal, la diarrea y la constipación, siendo uno de los principales, la esofagitis que se evita indicando al paciente que no se coloque en posición horizontal luego de la toma. Debido a que esta droga fue fue aprobada por la FDA en 1995 se desconocen sus efectos a largo plazo.

En el FIT (Fracture intervention trial), el alendronato demostró disminuir en un $50 \%$ el riesgo de fractura de cuello de fémur, muñeca y vértebras, observándose el mayor impacto en las pacientes con mayor riesgo de osteoporosis. En las pacientes que no habían tenído fracturas previamente el alendronato mostró una reducción absoluta del riesgo de fracturas del $1 \%$ ( $2 \%$ en el grupo placebo y $1 \%$ en el grupo alendronato), lo que nos habla de un NNT de 100 durante tres años. En cambio, en las pacientes que habían tenido fracturas previamente, la reducción absoluta del riesgo fue de $4.6 \%$ (18.3 a un $13.6 \%$ ) siendo el NNT durante tres años en este subgrupo de 22.

\section{2-2) Risedronato}

Este análogo del pirofosfato tiene acción antiresortiva como los anteriores y demostró disminuir el riesgo de fracturas vertebrales y de huesos largos en pacientes con fracturas vertebrales previas con un mejor perfil de efectos adversos que el alendronato (ej. los digestivos). Su dosis usual es de $5 \mathrm{mg}$ y se administra con $1000 \mathrm{mg}$ de calcio. En el estudio de Harris y col. recibieron vitamina $D$ aquellas pacientes cuyos niveles de 25 -hidroxivitamina D habían sido bajos.

\section{2-3) Otros bifosfonatos}

El etidronato disminuye la incidencia de fracturas en las pacientes con reducción de la densidad ósea por densitometría y en las que tuvieron mínimas fracturas vertebrales diagnósticadas radiológicamente. Debe ser usado por periodos cortos porque altera la mineralización del hueso, habiéndose observado su efectividad solamente a los dos años.

El pamidronato ha mostrado algunos resultados promisorios en pacientes con osteoporosis inducida por corticoides, menopausia precoz o inmovilización prolongada.

\section{3) Vitamina $D$}

Como se mencionó, en los gerontes el déficit de vitamina $D$ es el principal responsable de la pérdida ósea debido al hiper- paratiroidismo secundario. En estos pacientes se puede indicar vitamina D como suplemento vitamínico tal cual fue explicado previamente. Hay evidencia que la vitamina D3 a dosis de 0.25 a $0.50 \mathrm{mg} /$ día con suplemento de calcio puede ser utilizada principalmente en gerontes con déficit o en pacientes con osteoporosis secundaria al uso de corticoides.

\section{4) Calcitonina}

La calcitonina es otro fármaco antirresortivo que a dosis de $100 U I /$ día y asociada al calcio demostró mejorar el calcio corporal total, probablemente por mejorar el recambio óseo. Existen pequeños trabajos aleatorizados que demuestran disminución de la incidencia de fracturas vertebrales con su uso, siendo una buena opción terapéutica en pacientes con dolor ya que tiene un efecto analgésico. Sus desventajas son su costo elevado y el desconocimento que.poseemos acerca de su eficacia en el largo plazo.

\section{5) Fluoruros}

Los fluoruros estimulan la síntesis ósea por parte de los osteoblastos y mejoran notablemente la densidad mineral ósea. Sin embargo, aún no están aprobadas para el tratamiento de la osteoporosis. Un estudio mostró un aumento de las fracturas de cuello de fémur en pacientes que utilizaron fluoruros, se cree que este efecto se debe a que la droga altera la síntesis de hueso cortical disminuyendo la fuerza del hueso. Hay trabajos en los que se indicaron fluoruros a menor dosis y disminuyó el número de fracturas vertebrales.

\section{6) Raloxifeno}

El raloxifeno es un modulador selectivo de los receptores estrogénicos que produce los efectos de estas hormonas en los lípidos y en el hueso y los antagoniza (efecto antiestrogénico) en el tejido endometrial y mamario. Fue aprobado por la FDA en 1997 para la prevención de la osteoporosis.

El estudio multicéntrico, randomizado y aleatorizado conocido por La sigla MORE (Multiple Outcome of Raloxifene Evaluation) duró tres años e incluyó mujeres con osteoporosis vertebral definida por DMO (según criterios de OMS) o con fracturas definidas por radiografías. Las pacientes de ambos grupos recibieron $500 \mathrm{mg}$ de calcio y 400 a 600 UI de colecalciferol y fueron aleatorizadas a raloxifeno (60 y $120 \mathrm{mg}$ ) o a placebo. Luego de tres años de seguimiento, las que recibieron raloxifeno (a ambas dosis) tuvieron menos fracturas vertebrales $(0.6 \%)$, comparadas con las del grupo placebo $(1.4 \%)$, lo que habla de una reducción del riesgo relativo (RRA) de $0.8 \%$ y un número necesario para tratar (NNT) de 123. También mejoraron la densitometría y el colesterol total.

Algunas revisiones consideran que esta droga, además, reduce significativamente el colesterol LDL. En cuanto a su efecto sobre la proliferación endometrial, parece no estimularla, necesitándose más estudios para confirmar este punto. El raloxifeno, aparentemente, tampoco produce proliferación de las células mamarias, lo que lo convertiría en una buena alternativa para las mujeres con riesgo aumentado de cáncer de mama.

La dosis usual es de $60 \mathrm{mg}$ por día, acompañada con calcio y vitamina D. Los efectos adversos más comúnes son las tuforadas y los calambres de los miembros inferiores, siendo el de mayor relevancia clínica el aumento de los eventos tromboembólicos ( $1 \%$ vs. $0,3 \%$ en el grupo placebo) necesitándose tratar 143 pacientes durante tres años para que aparezca un evento adicional.

El cuadro 1 uno resume las recomendaciones según las diferentes situaciones clínicas. 


\section{CUADRO 1: CONDUCTAS RECOMENDADAS PARA LA PREVENCION Y EL TRATAMIENTO DE LA OSTEOPOROSIS(\#)}

¥Prevención primaria en todas las mujeres menopausicas: indicar actividad fisica y dieta rica en calcio con eventual suplemento, evitar el tabaco y el alcohol, informar sobre TRH y realizar prevención sobre caídas.

¥ Pacientes con osteopenia diagnosticada DMO (T entre -1 y -2.5 ): reforzar los tratamientos preventivos y evaluar indicación de TRH. En las mujeres de muy alto riesgo (aquellas con dos o más factores asociados, se recomienda comenzar precozmente con TRH o alendronato).

¥ Pacientes con osteoporosis diagnosticada por DMO (T menor a -2.5) y sin evidencias de fractura: indicar tratamiento para osteoporosis. Siempre que se pueda, se recomienda comenzar con TRH. Si ésta está contraindicada o es rechazada por la paciente, se pueden utilizar bifosfonatos. En ambos casos se recomienda asociar calcio y vitamina D. Reforzar tratamientos preventivos.

¥ Pacientes con evidencias clínicas (dolor) o radiográficas de fractura vertebral: la mayoría de las pacientes con fracturas vertebrales no han tenido síntomas en el momento de la fractura y ésta se encuentra como un hallazgo radiológico. La paciente con dolor inicialmente deberá hacer reposo y tomar analgésicos. Se le debe informar que en la fractura vertebral el dolor dura aproximadamente seis semanas. Una opción para el tratamiento del dolor es el uso de calcitonina. Se recomienda la terapia física en forma de caminatas o natación y la rehabilitación kinesiológica para disminuir las contracturas. Puede ser necesario el uso de un soporte para la columna (corset). El tratamiento farmacológico es indispensable. Debe recibir un aporte de calcio de $2 \mathrm{~g}$ por día y $400 \mathrm{a}$ 800 UI de vitamina D por día. Si se puede y la paciente lo acepta, se recomienda TRH, también se pueden usar bifosfonatos (mostraron reducir la incidencia de nuevas fracturas vertebrales en mujeres ya fracturadas). Reforzar los tratamientos preventivos.

¥ Pacientes con fractura de cadera: El tratamiento básico es la cirugía, luego de la cual los pacientes deberían recibir TRH más calcio a dosis de 1.5 a 2 gramos por día más vitamina D ó D3. Los bifosfonatos (ej. alendronato) podrían ser una alternativa. Reforzar los tratamientos preventivos y profundizar en la prevención de caídas (ej. realizando visitas al hogar para prevenir accidentes), recomendar el uso de lentes, de asientos para baño y evitar drogas que produzcan hipotensión ortostática y/o sedantes. En pacientes inmovilizados es importante colocar protección en forma de almohadillas en la región de la cadera.

\# Pacientes con fractura de cadera: El tratamiento básico es la cirugía, luego de la cual los pacientes deberían recibir TRH más calcio a dosis de 1.5 a 2 gramos por día más vitamina D ó D3. Los bifosfonatos (ej. alendronato) podrían ser una alternativa. Reforzar los tratamientos preventivos y profundizar en la prevención de caídas (ej. realizando visitas al hogar para prevenir accidentes), recomendar el uso de lentes, de asientos para baño y evitar drogas que produzcan hipotensión ortostática y/o sedantes. En pacientes inmovilizados es importante colocar protección en forma de almohadillas en la región de la cadera.

(\#) Es importante destacar que todos los tratamientos mencionados están bajo constante revisión en estudios controlados y aleatorizados.

\section{ALGUNAS REFLEXIONES RESPECTO DE LA NUEVA EVIDENCIA}

El descubrimiento de los fármacos con efecto modulador selectivo de los receptores estrogénicos como el raloxifeno y el avance de los bifosfonatos aportan nuevas estrategias para el tratamiento y la prevención de la osteoporosis.

Si pensamos en la indicación de la terapia preventiva o de tratamiento de la osteoporosis en el contexto global de las pacientes que pueden o no tener otros problemas de salud y/o factores de riesgo surgen las siguientes reflexiones:

Consideramos que en las mujeres que poseen un riesgo elevado de fracturarse y se encuentran cursando sintomáticamente la etapa temprana de la menopausia el tratamiento hormonal es la alternativa de elección, teniendo en cuenta que que es la menos costosa y recordando que los beneficios óseos de la TRH desaparecen cuando se suspende su uso.

En mujeres añosas surge como alternativa interesante el uso de bifosfonatos y en las que tengan riesgo aumentado de desarrollar cáncer de mama, el raloxifeno.

La droga ideal para ser usada en las mujeres sería aquella que tuviera efectos similares a los estrógenos en el corazón y en los huesos, que evitara los síntomas vasomotores y la atrofia urogenital y que actuara como antiestrógeno en la mama y en el endometrio. Por lo que sabemos, el raloxifeno se aproxima bastante a esta conjunto de características. Sin embargo, no debemos olvidar que provoca calores, tiene efecto antiestrogénico en aparato urogenital, aumenta el riesgo de fenómenos tromboembólicos y que aún no se ha demostrado que tenga acción preventiva sobre la enfermedad coronaria.

\section{Interrogantes que persisten}

Aún carecemos de evidencia sobre los efectos de las drogas a muy largo plazo ya que solamente conocemos los aspectos de la TRH y algo sobre los bifosfonatos.

Actualmente el seguimiento del tratamiento de la osteoporosis se realiza mediante una DM0 anual para evaluar la respuesta al mismo. En los casos en los que la respuesta es escasa o nula (DMO sin mejoría o que empeora) se sugiere una consulta con el especialista en metabolismo fosfocálcico.

Esta conducta es controvertida ya que todavía no está claro el verdadero impacto de que una densitometría no cambie, mejore o empeore o si hay medidas que reduzcan las fracturas de cuello independiente de la mejoría de los valores de la densitometría ósea. Por otro lado, la confiabilidad de la DMO es discutible y no se conoce claramente el grado de variabilidad que puede presentar cada método.

Si bien hoy contamos con distintas alternativas para el manejo de esta importante patología, la elección definitiva deberá basarse en la información y discusión de las opciones con nuestros pacientes. Para esto será imprescindible contar con estudios del mejor diseño posible que evaluén el efecto del ejercicio, de la administración de calcio, vitamina D, etc.; así como los que comparen la eficacia, efectividad y seguridad de los distintos tratamientos hoy disponibles entre sí.

\section{Dra. Claudia Dreyer}

Unidad de Medicina Familiar y Preventiva del Hopital Italiano de Buenos Aires

\section{Bibliografía recomendada}

Black DM, Cummings SR, Karpf DB, et al. Randomised trial of effect of alendronate on risk of fracture in women with existing vertebral fractures. Lancet 1996;348:1535-1541.

Black DM, et al. Randomised trial of effect of alendronate on risk of fracture in women with existing vertebral fractures. Fracture Intervention Trial Research Group. Lancet 1996; 348(9041): 1535-41.

Chapuy MC. et al. Vitamin D3 and calcium to prevent hip fractures in elderly women. N Engl J Med 1992: 327(23): 1637-42.

Compston JE, et al. Bone densitometry in clinical practice. BMJ 1995; 310(6993): 1507-10.

Discacciatti V. Monitoreo de la osteoporosis con densitometria mineral osea. Cuando cambiar de droga? EVIDENCIA en atención primaria. Año 2000. Numero 3, pag 77.

Effects of hormone therapy on bone mineral density: results from the postmenopausal estrogen/progestin interventions (PEPI) trial. The Writing Group of the PEPI. JAMA 1996; 276(17): 1389-96.

Ettinger B, Black DM, Mitlak BH. Reduction of vertebral Fracture Rish in Pot menopausal Womwn With 0steoporosis Treated With Raloxifene. Resultas From a 3 Years Randomised Clinical Trial. JAMA 1999:282:637-45. Harris S, Watts N, Nelson B, Paul D; for the Vertebral Efficacy With Risedronate Therapy (VERT) Group. Effects of Risedronate Treatment on Vertebral and Novertebral Fractures in Women With Menopausal Osteoporosis: A Randomized Controlled Trial. JAMA 1999;282:1344-1352.

Khovidhunkit W, Shoback D. Clinical Effects of raloxifene Hydrochloride in Women. Ann Intern Med 1999 ;130:431-439.

Liberman UA, Weiss SR, Broll J, et al. Effect of oral alendronate on bone mineral density and the incidence of fractures in postmenopausal osteoporosis. N Enql J Med 1995;333:1437-1443.

National Osteoporosis Foundation. Osteoporosis: Review of Evidence for Prevention, Diagnosis, and Treatment and Cost Effectiveness Analysis.

Scott J, da Camara C, Early J. Raloxifene : a Selective Estrogen receptor Modulator. Am Fam Physician 1999;60:1131-9. 